\title{
Aufgaben
}

\section{Neue Aufgaben}

Lösungen sind bis zum 10. November 2013 erbeten und können auf postalischem Weg an Dr. Stefan Grieder, Im eisernen Zeit 55, CH-8057 Zürich

gesandt werden. Lösungen, die in einem gängigen Format abgefasst sind, können als Attachment auch über die E-Mail-Adresse stefan.grieder@hispeed.ch eingereicht werden.

Aufgabe 1314: Man beweise für alle $x, y, z \in \mathbb{R}^{+}$mit $x+y+z=1$ und für alle $a \in[0,2]$ die Ungleichung

$$
0 \leq x y+y z+z x-a x y z \leq \frac{9-a}{27} .
$$

Dieter Rüthing, Paderborn, D

Aufgabe 1315: Es sei $f: \mathbb{R} \rightarrow \mathbb{R}$ die Funktion

$$
f(x)=\sum_{j=k}^{k l} c_{j}(k, l)\left(\begin{array}{l}
x \\
j
\end{array}\right)
$$

mit $k, l \in \mathbb{N}$ und den Koeffizienten

$$
c_{j}(k, l)=\sum_{i=k}^{j}(-1)^{j-i}\left(\begin{array}{l}
j \\
i
\end{array}\right)\left(\begin{array}{l}
i \\
k
\end{array}\right)^{l} .
$$

Man zeige, dass $f(x)=\left(\begin{array}{l}x \\ k\end{array}\right)^{l}$ gilt.

Friedhelm Götze, Jena, D

Aufgabe 1316 (Die einfache dritte Aufgabe): Die Winkelhalbierenden der Winkel $\beta$ und $\gamma$ des Dreiecks $A B C$ schneiden die Seitenhalbierende $A A^{\prime}$ in den Punkten $B^{\prime}$ und $C^{\prime}$. Zeige, dass aus $B B^{\prime}=C C^{\prime}$ die Gleichheit $A B=A C$ folgt.

Sadi Abu-Saymeh und Mowaffaq Hajja, Irbid, JOR 


\section{Lösungen zu den Aufgaben in Heft 2, 2012}

Aufgabe 1302. Mit der Rekursionsformel $f_{n+1}(x)=x f_{n}(x)-f_{n-1}(x)$ und $f_{0}(x)=$ $1, f_{1}(x)=x$ werden Polynome $f_{n}(x)$ vom Grad $n$ definiert. Man bestimme die Lösungen der Gleichung $f_{n}(x)=f_{n-1}(x)$.

André Ammann, Yverdon, $\mathrm{CH}$ und Lucien Pianaro, Annecy-le-Vieux, F

Auswertung der eingesandten Lösungen. Es sind 14 Zuschriften eingetroffen: Hans Brandstetter (Wien, A), Henri Carnal (Bern, CH), Friedhelm Götze (Jena, D), Frieder Grupp (Schweinfurt, D), Walther Janous (Innsbruck, A), Paul Jolissaint (Porrentruy, CH), Joachim Klose (Bonn, D), Stephan Kocher (Oberschrot, CH), Fritz Siegerist (Küsnacht, CH), François Sigrist (Neuchâtel), Jürgen Spilker (Freiburg, D), Michael Vowe (Therwil, $\mathrm{CH}$ ), Paul Weisenhorn (Achern, D) und Roland Wyss (Flumenthal, $\mathrm{CH}$ ).

Viele Löser bemerken die Verwandtschaft der Polynome $f_{n}$ mit den Tschebyscheff-Polynomen zweiter Art und verwenden dann Eigenschaften jener Polynome. Wir folgen der Lösung von Roland Wyss.

Die Differenzen $d_{n}(x)=f_{n}(x)-f_{n-1}(x)$ erfüllen die gleiche Rekursionsformel mit den Anfangsbedingungen $d_{0}(x)=x, d_{1}(x)=x-1$. Mit der Substitution $x=2 \cos (t)(0<$ $t<\pi)$ und dem üblichen Ansatz $d_{n}=z^{n}$ zum Lösen von linearen Differenzengleichungen hat man die charakteristische Gleichung

$$
z^{2}-2 z \cos (t)+1=0
$$

mit den Lösungen $e^{i t}$ und $e^{-i t}$.

Für die allgemeine Lösung $d_{n}=c_{1} e^{i n t}+c_{2} e^{-i n t}$ errechnet man geduldig die Koeffizienten $c_{1}, c_{2}$ aus den Anfangsbedingungen $c_{1}+c_{2}=1$ und $c_{1} e^{i t}+c_{2} e^{-i t}=2 \cos (t)-1$ und gewinnt so

$$
d_{n}=\frac{\sin ((n+1) t)-\sin (t)}{\sin (t)}=2 \frac{\sin \left(\frac{t}{2}\right)}{\sin (t)} \cos \left(\frac{2 n+1}{2} t\right) .
$$

Wegen $0<t<\pi$ hat man bloss das Verschwinden des zweiten Faktors zu studieren. Mit

$$
t_{k}=\frac{2 k+1}{2 n+1} \pi, \quad k=0,1,2, \ldots, n-1
$$

hat man für $n \geq 1$ die gefragten Lösungen

$$
x_{k}=2 \cos \left(\frac{2 k+1}{2 n+1} \pi\right), \quad k=0,1,2, \ldots, n-1
$$

der Aufgabensteller.

Aufgabe 1303. Es sei $2 \leq k \leq 9$.

a) Die Summe von $k$ aufeinanderfolgenden Quadratzahlen soll eine Dreieckszahl sein.

b) Die Summe von $k$ aufeinanderfolgenden Dreieckszahlen soll eine Quadratzahl sein. 
In beiden Fällen sind diejenigen Werte von $k$ gesucht, für die es unendlich viele Lösungen gibt. Die Lösungen sind dann rekursiv zu beschreiben.

Jany C. Binz, Bolligen, CH

Auswertung der eingesandten Lösungen. Folgende 9 Leser haben Beiträge eingesandt: Hans Brandstetter (Wien, A), Henri Carnal (Bern, CH), Friedhelm Götze (Jena, D), Frieder Grupp (Schweinfurt, D), Walther Janous (Innsbruck, A), Fritz Siegerist (Küsnacht, $\mathrm{CH}$ ), Jürgen Spilker (Freiburg, D), Michael Vowe (Therwil, CH) und Paul Weisenhorn (Achern, D).

Wir folgen der Lösung von Henri Carnal.

a) Aus $S_{n}=1^{2}+2^{2}+\cdots+n^{2}=\left(2 n^{3}+3 n^{2}+n\right) / 6$ folgt

$$
S_{n+k}-S_{n}=k n(n+k+1)+S_{k}=k \frac{(2 n+k+1)^{2}-(k+1)^{2}}{4}+S_{k}
$$

Ist dies eine Dreieckszahl $\left(\begin{array}{c}m \\ 2\end{array}\right)=\left[(2 m-1)^{2}-1\right] / 8$, so hat man (für ein festes $k$ und mit $u=2 m-1, v=2 n+k+1)$ die Pellsche Gleichung

$$
P: u^{2}-2 k v^{2}=8 S_{k}-2 k(k+1)^{2}+1=\frac{2}{3} k\left(k^{2}-1\right)+1
$$

zu lösen.

$k=2: \quad P$ ergibt $u^{2}-4 v^{2}=5$ mit der einzigen Lösung $u=3, v=1$.

$k=3: u^{2}-6 v^{2}=17 \equiv_{3} 2$ hat keine Lösung $\left(u^{2} \equiv_{3} 0\right.$ oder 1$)$.

$k=4: \quad u^{2}-8 v^{2}=41$ hat die Grundlösung $\left(u_{0}, v_{0}\right)=(7, \pm 1)$. Die quadratische Form $u^{2}-8 v^{2}$ ist bei der (umkehrbaren) Transformation mit Matrix $A$

$$
\left(\begin{array}{l}
u^{\prime} \\
v^{\prime}
\end{array}\right)=\left(\begin{array}{ll}
3 & 8 \\
1 & 3
\end{array}\right)\left(\begin{array}{l}
u \\
v
\end{array}\right)
$$

invariant, sodass $\left(u^{\prime}, v^{\prime}\right)$ wieder eine Lösung ist. Allerdings ist dann $v^{\prime}$ gerade und damit unzulässig $(v=2 n+5)$. Man muss also $A$ durch $B=A^{2}=\left(\begin{array}{cc}17 & 48 \\ 6 & 17\end{array}\right)$ ersetzen. Da $B^{2}=34 B-I$ gilt, erhält man die Rekursionsformel

$$
\left(u_{i+2}, v_{i+2}\right)=34\left(u_{i+1}, v_{i+1}\right)-\left(u_{i}, v_{i}\right)
$$

und zwei Folgen mit $\left(u_{1}, v_{1}\right)=(71,25)$ bzw. $(167,59)$.

$k=5: \quad u^{2}-10 v^{2}=81$ hat die Grundlösungen $\left(u_{0}, v_{0}\right)=(9,0),(11, \pm 2)$ sowie $(21, \pm 6)$. Die Übergangsmatrix ist $\left(\begin{array}{cc}19 & 60 \\ 6 & 19\end{array}\right)$ und die Rekursionsformel

$$
\left(u_{i+2}, v_{i+2}\right)=38\left(u_{i+1}, v_{i+1}\right)-\left(u_{i}, v_{i}\right) \quad(5 \text { Folgen }) .
$$

$k=6: \quad u^{2}-12 v^{2}=141=3 \cdot 47$ führt zu $u=3 w, 3 w^{2}-4 v^{2}=47$ mit den Grundlösungen $\left(w_{0}, v_{0}\right)=(7, \pm 5)$, der Matrix $\left(\begin{array}{ll}7 & 8 \\ 6 & 7\end{array}\right)$ und der Formel

$$
\left(w_{i+2}, v_{i+2}\right)=14\left(w_{i+1}, v_{i+1}\right)-\left(w_{i}, v_{i}\right) \quad(2 \text { Folgen }) .
$$


$k=7: \quad u^{2}-14 v^{2}=225$ führt zu $u^{2} \equiv 32 v^{2} \Rightarrow 3|u, 3| v$ also $u=3 w, v=$ $3 x, w^{2}-14 x^{2}=25$. Grundlösungen $\left(w_{0}, x_{0}\right)=(5,0)$, sowie $(9, \pm 2)$, Matrix $\left(\begin{array}{cc}15 & 56 \\ 4 & 15\end{array}\right)$, Formel

$$
\left.\left(w_{i+2}, x_{i+2}\right)=30\left(w_{i+1}, x_{i+1}\right)-\left(w_{i}, x_{i}\right) \quad \text { (3 Folgen }\right) .
$$

$k=8: \quad u^{2}-16 v^{2}=337$ (Primzahl) führt zu $u=169, v=42$ (als gerade Zahl ist $v$ unzulässig).

$k=9: \quad u^{2}-18 v^{2}=481=13 \cdot 37$. Da $18 \equiv_{13} 5$ keine Quadratzahl mod 13 ist, müsste $13 \mid u$ und $13 \mid v$ gelten, also $13^{2} \mid 481$, was nicht der Fall ist. Daher gibt es keine Lösungen.

b) Es ist $T_{n}=\sum_{i=1}^{n}\left(\begin{array}{c}i \\ 2\end{array}\right)=\left(\begin{array}{c}n+1 \\ 3\end{array}\right)=\frac{n^{3}-n}{6}$, somit

$$
T_{n+k}-T_{n}=\frac{1}{2} k n(k+n)+T_{k}=\frac{k}{8}(2 n+k)^{2}+\frac{k^{3}-4 k}{24} .
$$

Ist dies gleich $m^{2}$, so hat man (mit $u=2 m, v=2 n+k$ )

$$
Q: 2 u^{2}-k v^{2}=\frac{k^{3}-4 k}{3} .
$$

Ist $k$ gerade $(k=2 h)$, so setzt man $w=n+h$ und erhält

$$
R: m^{2}-h w^{2}=\frac{h\left(h^{2}-1\right)}{3} .
$$

$k=2: \quad R$ ergibt $m^{2}=w^{2}=(n+1)^{2}$. Die Lösung $m=n+1$ existiert immer.

$k=4: \quad m^{2}-2 w^{2}=2$ hat die Grundlösung $\left(m_{0}, w_{0}\right)=(2,1)$, die Matrix $\left(\begin{array}{ll}3 & 4 \\ 2 & 3\end{array}\right)$ und die Formel

$$
\left(m_{i+2}, w_{i+2}\right)=6\left(m_{i+1}, w_{i+1}\right)-\left(m_{i}, w_{i}\right) \quad\left(\operatorname{mit}\left(m_{1}, w_{1}\right)=(10,7)\right) .
$$

$k=6: m^{2}-3 w^{2}=8 \equiv_{3} 2$ hat keine Lösung $\left(m^{2} \equiv_{3} 0\right.$ oder 1$)$.

$k=8: \quad m^{2}-4 w^{2}=20$ hat nur die Lösung $m=6, w=2$, somit $n=-2$.

$k=3: \quad Q$ ergibt $2 u^{2}-3 v^{2}=5$ mit den Grundlösungen $\left(u_{0}, v_{0}\right)=(2, \pm 1)$, der Matrix $\left(\begin{array}{ll}5 & 6 \\ 4 & 5\end{array}\right)$ und der Formel

$\left(u_{i+2}, v_{i+2}\right)=10\left(u_{i+1}, v_{i+1}\right)-\left(u_{i}, v_{i}\right) \quad\left(\operatorname{mit}\left(u_{1}, v_{1}\right)=(4,3)\right.$ bzw. $\left.(16,13)\right)$.

$k=5: 2 u^{2}-5 v^{2}=35$ bedingt $5 \mid u$, also $u=5 w, 10 w^{2}-v^{2}=7$ d.h. $v^{2} \equiv_{10} 3$. Keine Lösung.

$k=7: \quad 2 u^{2}-7 v^{2}=105$ bedingt $7 \mid u$, also $u=7 w, 14 w^{2}-v^{2}=15$ d.h. $v^{2} \equiv_{7}-1$. Keine Lösung.

$k=9: \quad 2 u^{2}-9 v^{2}=231=3 \cdot 77$ bedingt $3 \mid u, u=3 w$. Dies ist unmöglich, weil $9 \nmid 231$.

Aufgabe 1304 (Die einfache dritte Aufgabe). In Restaurants wird um den Stiel eines Tulpenglases oft ein aufgeschnittener Kreisring mit den Radien $r, R(0<r<R)$ aus 
saugfähigem Papier gelegt. Dieses kann man zum Spielen entfernen und durch Überlappen der Papierschnittlinien zu einem Kreiskegelstumpf verformen.

Wie gross wird die Höhe des Kegelstumpfes in Abhängigkeit der Überlappungslänge $u(0 \leq u<2 \pi R)$ gemessen auf der äusseren Kreislinie des Papiers?

Roland Wyss, Flumenthal, $\mathrm{CH}$

Auswertung der eingesandten Lösungen. Es sind Lösungen von 8 Lesern eingetroffen: Jany C. Binz (Bolligen, CH), Hans Brandstetter (Wien, A), Henri Carnal (Bern, CH), Hans Egli (Zürich, CH), Frieder Grupp (Schweinfurt, D), Fritz Siegerist (Küsnacht, CH), Michael Vowe (Therwil, CH), Paul Weisenhorn (Achern, D) und 5 Lösungen der Bündner Kantonsschule, Klasse 5, Schwerpunkt Physik und Anwendungen der Mathematik (Chur, $\mathrm{CH}$ ): Benjamin Arner (Soazza, CH), Janik Feiner (Chur, CH), Moritz Hoff (Tamins, CH), Gian Jörimann (Flims, CH), Rajika Kurumoorthy (Chur, $\mathrm{CH}$ ), Djurdjina Pavlovic (Chur, $\mathrm{CH}$ ), Marco Pires (Tamins, $\mathrm{CH}$ ), Marco Ruggia (Chur, $\mathrm{CH}$ ), Laurin Schwitter (Masein, $\mathrm{CH}$ ), Matthias von Blumenthal (Rodels, $\mathrm{CH}$ ), Stefano Weidmann (Chur, $\mathrm{CH}$ ) und Armon Zimmermann (Tamins, $\mathrm{CH}$ ).

Alle Löser gingen mehr oder wenig gleich vor wie Frieder Grupp, dessen Lösung wir hier präsentieren.
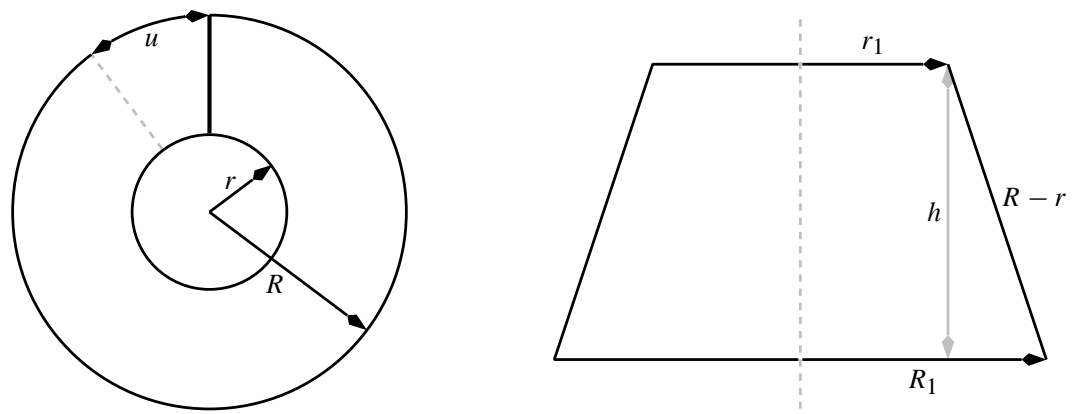

Wir betrachten zunächst den ebenen Kreisring. Ist $u$ die Überlappungslänge auf der äusseren Kreislinie, so ist $\frac{r}{R} u$ die Überlappungslänge auf der inneren Kreislinie. Sind $r_{1}<R_{1}$ die Radien nach der Überlappung, so gilt

$$
2 \pi R_{1}=2 \pi R-u, \quad 2 \pi r_{1}=2 \pi r-\frac{r}{R} u
$$

und es folgt (senkrechter Schnitt durch den Kegelstumpf)

$$
\left(R_{1}-r_{1}\right)^{2}+h^{2}=(R-r)^{2},
$$

wobei $h$ die Höhe des Kegelstumpfes ist. Insgesamt erhält man

$$
h^{2}=(R-r)^{2}-\left((R-r)-\frac{u}{2 \pi}\left(1-\frac{r}{R}\right)\right)^{2}=\frac{u}{\pi R}(R-r)^{2}\left(1-\frac{u}{4 \pi R}\right),
$$

also

$$
h=(R-r) \sqrt{\frac{u}{\pi R}\left(1-\frac{u}{4 \pi R}\right)} .
$$

